\title{
Shared Genetic Factors in the Co-Occurrence of Depression and Fatigue
}

\author{
Elizabeth C. Corfield, ${ }^{1}$ Nicholas G. Martin, ${ }^{2}$ and Dale R. Nyholt ${ }^{1}$ \\ ${ }^{1}$ Faculty of Health, Institute of Health and Biomedical Innovation, Queensland University of Technology, Brisbane, Australia \\ ${ }^{2}$ QIMR Berghofer Medical Research Institute, Brisbane, Queensland, Australia
}

\begin{abstract}
Depression and fatigue have previously been suggested to share an underlying genetic contribution. The present study aims to investigate and characterize the familiality and genetic relationship between depression and fatigue. The familiality of depression and fatigue was assessed by calculating relative risks, measured by the prevalence ratio, within 643 monozygotic (MZ) and 577 dizygotic (DZ) twin pairs. Bivariate twin modeling was utilized to assess the magnitude of shared heritability between depression and fatigue. Finally, the relationship between depression and fatigue was investigated using the co-twin control method, to determine whether the association is explained by causal or non-causal models. We observed an increased risk of fatigue in co-twins of probands with depression and increased risk of depression in cotwins of probands with fatigue. Higher risks were observed in MZ compared to DZ twin pairs, and bivariate heritability analyses indicated significant genetic components for depression and fatigue, with heritability estimates of $48 \%$ and $41 \%$, respectively. Importantly, a significant additive genetic correlation of 0.71 [95\% $\mathrm{Cl}=0.51-0.92)$ and bivariate heritability of $21 \%[95 \% \mathrm{Cl}=10-35 \%]$ was observed between depression and fatigue. Furthermore, results from the co-twin control method indicate a non-causal genetic relationship that likely explains the association between depression and fatigue. Notably, the contribution of shared genetic factors remained significant, independent of the overlapping symptoms, indicating that the relationship between co-occurring depression and fatigue is primarily due to shared genetic factors rather than overlapping symptomatology.
\end{abstract}

Keywords: fatigue, depression, heritability, genetics, twin study, comorbidity, relationship

Depression and fatigue are highly prevalent traits and are associated with a considerable reduction in quality of life. The genetic heritability of depression and fatigue has been estimated to range from $17 \%$ to $78 \%$ and $18 \%$ to $51 \%$, respectively (Schur et al., 2007; Sullivan et al., 2000; Sullivan et al., 2005). The wide ranges are likely attributable to differences in ethnicity and gender distribution within the study populations. Shared genetic etiologies have been implicated in studies investigating the heritability of psychological distress, anxiety, depression, and fatigue; and insomnia, fatigue, and depression (Hickie et al., 1999; Hur et al., 2012). However, the underlying mechanisms associated with depression and fatigue that could explain the high levels of comorbidity are poorly understood.

Two studies have tested for a shared genetic influence to depression and fatigue. In the first study, the heritability of lifetime-ever disabling fatigue (assessed by parental report using the disabling fatigue measure; Farmer et al., 1999) and depression within the past 3 months (assessed by the mood and feelings questionnaire in individuals over
11 years; Costello \& Angold, 1988) was investigated in children (aged 8-17; Fowler et al., 2006). The second study examined the genetic relationship between abnormal fatigue (assessed by the Chalder Fatigue Questionnaire; Chalder et al., 1993) and an indicator of lifetime-ever depression (assessed by two screening questions of the Composite International Diagnostic Interview; World Health Organization, 1990) regarding depressed mood and loss of interest - the two core symptoms of a major depressive episode, as defined by the Diagnostic and Statistical Manual of Mental Disorders (5th ed.; DSM-5; American Psychiatric Association, 2013) - in a Sri Lankan population (aged $\geq 15$

RECEIVED 25 May 2016; ACCEPTED 15 August 2016. First published online 5 October 2016.

ADDRESS FOR CORRESPONDENCE: Elizabeth C. Corfield, Institute of Health and Biomedical Innovation, Queensland University of Technology, GPO Box 2434, Brisbane QLD, 4001, Australia. E-mail: elizabeth.corfield@hdr.qut.edu.au 
years; Ball et al., 2010). Although both studies indicated depression and fatigue have a shared genetic contribution, the genetic relationship between co-occurring depression and fatigue is not well characterized and their results are not readily comparable due to the fact that risk for depression differs by age and sex (Bijl et al., 2002; Centers for Disease Control and Prevention, 2010; Kessler et al., 2003).

Differences in familial and genetic risk for depression have been identified with age. Older adults exhibit the lowest prevalence of a current depression diagnosis and a comparable risk of onset between males and females (Bebbington et al., 1998; Faravelli et al., 2013). Additionally, symptomatology differences have been observed between depression patients in different age ranges (Hybels et al., 2012; Wilkowska-Chmielewska et al., 2013). However, little is known about the genetic relationship between cooccurring depression and fatigue in older adults. Therefore, the present study utilizes relative risks (RR) and twin modeling to investigate the familiality and heritability of depression and fatigue within older adults. Furthermore, the cotwin control method was utilized to investigate whether the association between depression and fatigue is explained by a causal model or shared underlying etiology.

\section{Materials and Methods Study Cohort}

The present study was conducted using data from the over50s (aged) study conducted by the genetic epidemiology group within QIMR Berghofer. The study invited 2,281 twin pairs from the Australian Twin Registry to complete a mailed Health and Lifestyle Questionnaire (Bucholz et al., 1998; Mosing et al., 2012). The present study utilized responses to the Schedule of Fatigue and Anergia (SOFA), the 12-item General Health Questionnaire (GHQ), and the 14item Delusions Symptoms-States Inventory, States of Anxiety and Depression (DSSI/sAD) questionnaires (Bedford \& Deary, 1997; Goldberg \& Blackwell, 1970; Hickie et al., 1996). The study cohort utilized here overlaps the cohort used by Hickie et al. (1999) to investigate the multivariate heritability of psychological distress, anxiety, depression, and fatigue. However, the present study focuses on depression and fatigue, including looking at major depressive disorder (MDD) and minor depressive disorder MiDD.

\section{Diagnosis of Depression and Fatigue}

MDD and MiDD were classified using the nine criteria of a major depressive episode (depressed mood, anhedonia, a change in weight or appetite, insomnia or hypersomnia, psychomotor agitation or retardation, fatigue or loss of energy, feelings of worthlessness or excessive guilt, inability to concentrate or make decisions, and thoughts about death, suicidal thoughts, suicidal plans, or suicidal attempts), as defined by the DSM-IV criteria (American Psychiatric Association, 2000). A combination of questions from the GHQ and DSSI/sAD were used to assess depression, through assignment of specific questions to the appropriate criterion of the major depressive episode criteria. When multiple questions assessed a criterion, at least one positive response indicated the individual exhibited a symptom from the specific criterion. Each criterion was assessed by assigning 1 to the criterion if a symptom was exhibited by the individual and 0 if none of the symptoms for the criterion were met. The survey did not contain any assessment of change in weight or appetite; therefore, this criterion of a major depressive episode was not assessed. The scores of the remaining eight criteria assessed were summed, if the individual screened positive (score $>0$ ) for depressed mood and/or anhedonia; otherwise, the individual was assigned a total score of 0 . Individuals were classified as MDD, MiDD, or non-depressed, if they had a score of 5 or more, 2 to 4 , or less than 2, respectively.

The SOFA was originally designed to identify chronic fatigue syndrome cases. Therefore, physical, neurocognitive, and neurovegetative fatigue symptoms were assessed by the questionnaire. Consequently, the fatigued state identified by the SOFA is distinct from the fatigue experienced within a major depressive episode. Ten questions are contained in the SOFA; however, a shorter eight-item version was included in the survey due to two questions being replicated within the GHQ. Individuals were classified as fatigued if they reported 3 or more of the 10 fatigue symptoms (muscle pain at rest, post-exertional muscle pain, post-exertional muscle fatigue, post-exertional fatigue, hypersomnia, insomnia, poor concentration, speech problems, poor memory, and headaches).

\section{Familial Clustering}

Familiality between depression and fatigue was assessed by the calculation of $\mathrm{RR}$, assessed by the prevalence ratio, with their 95\% confidence intervals (CI). Initially, crosstabulation was utilized to assess the depression and fatigue status within twin pairs, based on zygosity groupings. The method by Nyholt and colleagues (2004) was utilized to estimate the risk within the complete cohort and same-sex twin pairs, where the cross-tabulations from using twin 1 or twin 2 as the proband were averaged. RR were also calculated from the averaged cross-tabulations within samesex monozygotic (MZ) and dizygotic (DZ) twin pairs and opposite-sex DZ twin pairs relative to non-depressed or non-fatigued status. Initially, the risk of fatigue in co-twins of depressed probands was calculated. Similarly, the risk of depression in co-twins of fatigued probands was calculated.

To assess the familiality of depression and fatigue independent of their overlapping symptoms, the risk of fatigue in co-twins was also estimated in the subgroup of depressed individuals without overlapping DSM depression symptoms (i.e., insomnia, poor concentration, and hypersomnia). Similarly, the risk of depression in co-twins was also estimated in the subgroup of fatigued individuals with- 
out overlapping fatigue symptoms (i.e., insomnia, inability to concentrate, and loss of energy).

\section{Genetic Analysis}

The association between depression and fatigue was assessed by looking at twin, phenotypic, and cross-twin, cross-trait correlations within MZ and DZ twin pairs. Polychoric correlations were calculated (due to the binary coding utilized within the cohort) using the polycor package in $\mathrm{R}$ (R Core Team, 2014). Twin correlations assessed the association of a single trait across a twin pair, phenotypic correlations assessed the association of two traits within individuals, and cross-twin, cross-trait correlations assessed the association of two traits across a twin pair. Twin and crosstwin, cross-trait correlations, which are larger in MZ compared to DZ twin pairs, indicate the etiology of the traits has a genetic contribution.

Bivariate twin models were calculated to estimate the relative contribution of genetic and environmental factors on the covariation of depression and fatigue. The bivariate twin modeling was conducted utilizing the Cholesky decomposition that allowed the genetic and environmental factors of the first trait to load onto the second trait (Neale \& Cardon, 1992). The model contains another set of genetic and environmental factors that are unique to the second trait. Such twin modeling partitions the observed phenotypic variance into specific components. Briefly, phenotypic differences between $M Z$ and $D Z$ twin pairs (MZ twin pairs have the same genotype and common environment while DZ twin pairs only share $50 \%$ of their genes but have the same common environment) are used to estimate the contribution of additive genetic (A), dominant (non-additive) genetic (D), common environmental (C), and unique environmental (E) variance components (Neale \& Cardon, 1992). Additionally, the genetic $\left(r_{\mathrm{g}}\right)$ and environmental $\left(r_{\mathrm{e}}\right)$ correlation between depression and fatigue was calculated as a measure of the overlap in gene and environmental sets, respectively.

Heritability estimates were calculated utilizing the structural equation modeling, including the threshold model. The threshold model posits that distinct traits represent a single, normally distributed, severity continuum. Initially, a single threshold was utilized for depression, whereby individuals were separated into a broad, two-category (nondepressed and MiDD/MDD) depression definition. Additionally, two thresholds were used to assess the threecategory (non-depressed, MiDD, and MDD) depression definition. A single threshold was used within all models to separate non-fatigued and fatigued individuals. Corrections for (linear) age and sex effects were included in all models, which were fitted using the OpenMx package in R (Boker et al., 2011; R Core Team, 2014). The significance of the variance components was assessed by comparing the fit of the full model (ACE/ADE) to the nested submodels (AE, $\mathrm{CE}$, and $\mathrm{E}$ ) where individual variance components were dropped from the model. The goodness-of-fit parameters used to assess the differences in the twin models were the likelihood-ratio chi-square test $\left(\chi^{2}\right)$, the difference in degrees of freedom $(\Delta d f)$, and $p$ value. Additionally, model fit was compared utilizing Akaike's Information Criteria (AIC) with the lowest AIC indicating the most parsimonious model (Akaike, 1973; 1974).

Polychoric correlations and bivariate heritability estimates were also estimated for depression and fatigue in the subgroup of twins without overlapping symptoms (i.e., insomnia, concentration problems, hypersomnia, and loss of energy).

\section{Relationship Analysis}

The co-twin control method was utilized to determine the type of relationship that exists between depression and fatigue (Kendler et al., 1993; Kendler et al., 1999). A causal relationship exists when a risk factor directly causes a phenotype, without familial confounding. Meanwhile, a noncausal model exists when familial (A and C) factors completely explain the correlation between the risk factor and the trait. Furthermore, non-causal relationships exist where the association between the risk factor and the trait is mediated by familial factors (A or C; Kendler et al., 1993; Kendler et al., 1999; McGue et al., 2010).

The co-twin control method conducted throughout the study followed the protocol outlined by Ligthart et al. (2010), where the odds ratio (OR) of the trait is calculated based on the presence or absence of the risk factor within three cohorts: $\mathrm{MZ}$ and $\mathrm{DZ}$ twin pairs with the trait that are discordant for the risk factor and a general population sample. The over-50s (aged) study contained $200 \mathrm{MZ}$ twin pairs and $215 \mathrm{DZ}$ twin pairs with a measure of depression that was discordant for fatigue. Similarly, $99 \mathrm{MZ}$ twin pairs and $96 \mathrm{DZ}$ twin pairs with a measure of fatigue were discordant for depression. The general population sample of 1,266 individuals was obtained by selecting all unpaired twin singles and randomly selecting a single individual from each complete twin pair (i.e., one individual from each family), excluding those discordant for fatigue or depression.

If a causal relationship exists between the risk factor and the trait of interest, the three cohorts are expected to have comparably elevated ORs (Figure 1). Similarly, under noncausal models, the general population is expected to show increased odds of exhibiting the trait, given the presence of the risk factor. However, MZ and DZ cohorts are expected to exhibit varying $O R$ patterns, although the association should always be smaller than within the general population. If the relationship between the risk factor and the trait is non-causal, no association is expected in the MZ cohort, whereas the DZ cohort is expected to exhibit a small association (Figure 1). Similarly, if a non-causal relationship exists between the risk factor and the trait that is mediated by shared environment, both the MZ and DZ cohorts are expected to exhibit a similar association (Figure 1). Finally, under a non-causal model mediated by genetic factors, the 


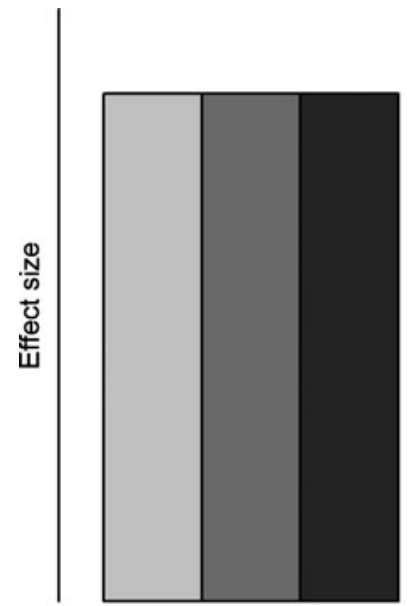

Causal

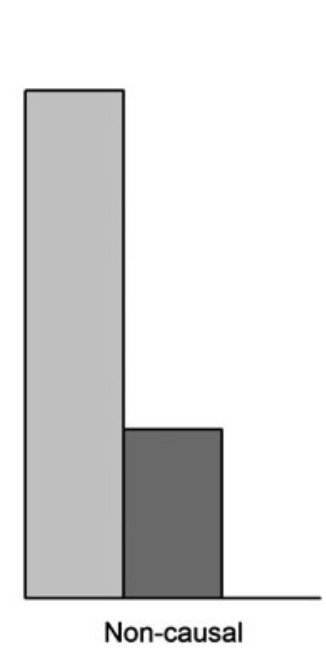

Non-causal

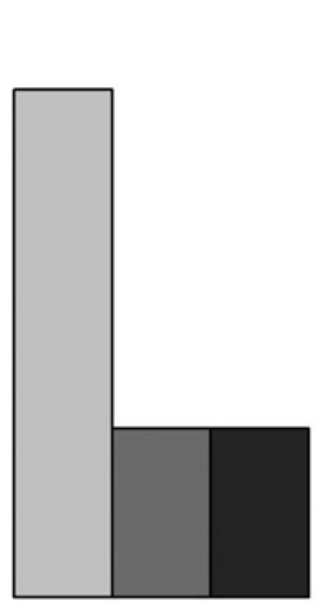

Non-causal shared environment
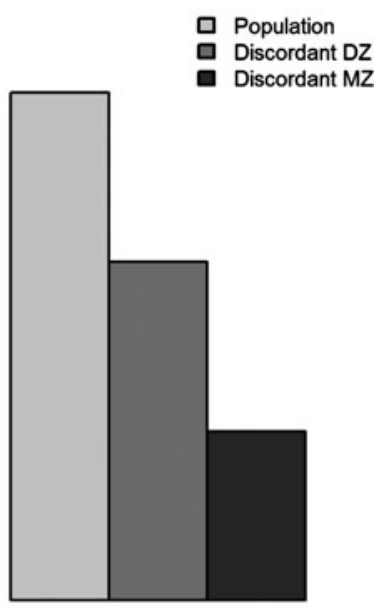

Non-causal genetic

\section{FIGURE 1}

Expected outcomes of the co-twin control method under the causal, non-causal, non-causal shared environment, and non-causal genetic models within the general population (light grey), discordant DZ twin pairs (grey) who share $50 \%$ of their genetics and $100 \%$ of their common environment, and discordant MZ twin pairs (dark grey) who share $100 \%$ of their genetics and common environment. Under a causal model, an association is expected within all three groups. Under a non-causal model, an association is expected within the general population, discordant DZ cohort will have a small association, and discordant MZ cohort will have no association. Similarly, under the non-causal shared environmental model, discordant DZ and MZ twin pairs have a small, equal association. Finally, under the non-causal genetic model, discordant DZ twin pairs have an association, whereas discordant MZ twin pairs have a smaller association.

MZ cohort is expected to exhibit a smaller association than the DZ cohort (Figure 1).

\section{Results}

The over-50s (aged) study was a population-based cohort of 2,281 Australian twin pairs. A total of 1,061 twin pairs were removed due to incomplete depression and fatigue classifications for one or both twins. The remaining 1,220 twin pairs consisted of $643 \mathrm{MZ}$ twin pairs (491 female-female and 152 male-male twin pairs) with a mean age of $61.5 \pm$ 8.9 (range $=50-92$ ) and 577 DZ twin pairs (263 femalefemale, 73 male-male, 136 female-male, and 105 malefemale twin pairs) with a mean age of $61.2 \pm 8.2$ (range = 50-90). The prevalence of depression (either MDD or MiDD) and fatigue was $11.0 \%$ (11.7\% of females, $9.3 \%$ of males) and $29.6 \%$ (32.5\% of females, $24.7 \%$ of males), respectively.

\section{Relative Risks}

Initially, all individuals who participated in the over-50s (aged) study were assessed for both MDD and MiDD (Supplementary Table S1). The present study focused on a twocategory, broad definition of depression defined as either MDD or MiDD. Cross-tabulation within MZ, same-sex DZ, and opposite-sex DZ twin pairs was based on depressed or non-depressed and fatigued or non-fatigued classifications (Table 1).

We observed an increased risk of fatigue in co-twins of probands with depression and increased risk of depression

\section{TABLE 1}

Cross-Tabulation a of Two-Category Depression and Fatigue Status Within Twin Pairs

\begin{tabular}{|c|c|c|c|c|}
\hline & & Non-depressed & Depressed & Total \\
\hline \multirow[t]{3}{*}{$M Z$} & Non-fatigued & 411 & 35 & 446 \\
\hline & Fatigued & 157.5 & 39.5 & 197 \\
\hline & Total & 568.5 & 74.5 & 643 \\
\hline \multirow[t]{3}{*}{$D Z_{s s}$} & Non-fatigued & 212 & 22.5 & 234.5 \\
\hline & Fatigued & 90.5 & 11 & 101.5 \\
\hline & Total & 302.5 & 33.5 & 336 \\
\hline \multirow[t]{3}{*}{$\mathrm{DZ}_{\mathrm{os}}[\mathrm{F}-\mathrm{M}]$} & Non-fatigued & 163 & 16 & 179 \\
\hline & Fatigued & 54 & 8 & 62 \\
\hline & Total & 217 & 24 & 241 \\
\hline \multirow[t]{3}{*}{$\mathrm{DZ}_{\mathrm{os}}[\mathrm{M}-\mathrm{F}]$} & Non-fatigued & 159 & 20 & 180 \\
\hline & Fatigued & 53 & 9 & 61 \\
\hline & Total & 212 & 29 & 241 \\
\hline
\end{tabular}

Note: $M Z=$ monozygotic; $D_{s s}=$ same-sex dizygotic; $D_{\mathrm{os}}=$ oppositesex dizygotic; $\mathrm{F}-\mathrm{M}=$ female-male; $\mathrm{M}-\mathrm{F}=$ male-female. ${ }^{\mathrm{a}}$ Tables were made symmetrical in same-sex twin pairs by averaging over using either twin 1 or twin 2 as proband. For example, within the complete twin pairs there were 155 twin pairs where twin 1 was fatigued and twin 2 was non-depressed and 160 twin pairs where twin 2 was fatigued and twin 1 was non-depressed. Therefore, the cross-tabulation averaging over twin 1 or twin 2 as proband is $(155+160) / 2=157.5$.

in co-twins of probands with fatigue, indicating a significant familial association between the traits (Table 2). Strong evidence for a genetic contribution is provided by the higher risk observed in $\mathrm{MZ}$ compared to DZ twin pairs. In particular, the risk of fatigue in co-twins of depressed probands was 1.91 [95\% CI $=1.49-2.46]$ in $\mathrm{MZ}$ twin pairs compared to $1.10[95 \% \mathrm{CI}=0.66-1.84]$ in same-sex DZ twin pairs. Similarly, the risk of depression in co-twins of fatigued probands 


\section{TABLE 2}

Relative Risk ${ }^{a}$ of Two-Category Depression and Fatigue Within Monozygotic, Same-Sex Dizygotic, and Opposite-Sex Dizygotic Twin Pairs

\begin{tabular}{|c|c|c|c|c|}
\hline Proband-co-twin & MZ(643 twin pairs) & $\mathrm{DZ}_{\mathrm{ss}}$ (336 twin pairs) & $\mathrm{DZ}$ os $[\mathrm{F}-\mathrm{M}](241$ twin pairs) & $D Z_{\text {os }}[M-F](241$ twin pairs $)$ \\
\hline Depressed-fatigued & $1.91(1.49-2.46)$ & $1.10(0.66-1.84)$ & $1.24(0.69-2.24)$ & $1.34(0.73-2.47)$ \\
\hline Fatigued-depressed & $2.56(1.67-3.90)$ & $1.13(0.57-2.23)$ & $1.44(0.65-3.21)$ & $1.30(0.62-2.70)$ \\
\hline
\end{tabular}
risks were calculated with respect to non-depressed or non-fatigued status in twin 1.

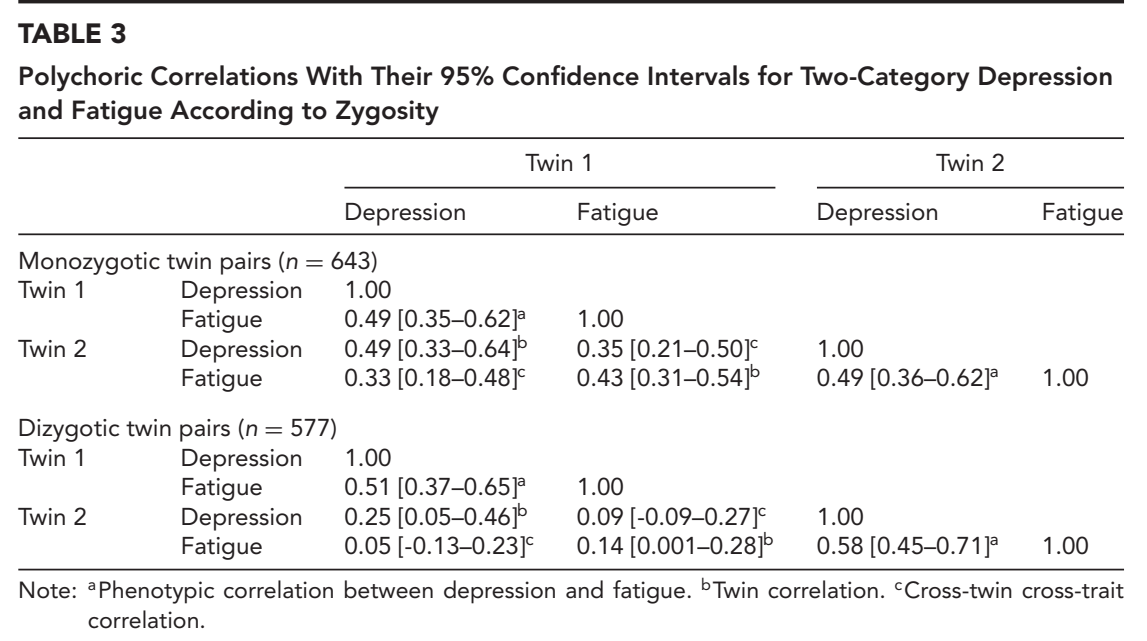

was $2.56[95 \% \mathrm{CI}=1.67-3.90]$ in $\mathrm{MZ}$ twin pairs compared to $1.13[95 \% \mathrm{CI}=0.57-2.23]$ in same-sex DZ twin pairs. Analysis of familial clustering within males and females indicated a similar pattern of risks (Supplementary Table S2). A comparable pattern of MZ to DZ RR was also observed for the separate MiDD and MDD cases, but with MDD producing further increased RR (Supplementary Table S3). Importantly, a similar pattern of risks was observed when they were estimated independently of depression and fatigue overlapping symptoms (Supplementary Tables S7S8).

\section{Polychoric Correlations}

The twin correlations for depression and fatigue were approximately two and three times larger in MZ compared to DZ twin pairs, respectively (Table 3). Similarly, the crosstwin, cross-trait correlations for depression and fatigue were over twice the magnitude in $\mathrm{MZ}$ compared to $\mathrm{DZ}$ twin pairs. These observed MZ $>\mathrm{DZ}$ correlations indicate that additive genetic factors contribute to the association between depression and fatigue. A similar pattern of polychoric correlations was observed when the analyses were repeated independently of the overlapping symptoms (Supplementary Table S9). Furthermore, comparable patterns of polychoric correlations were observed for MiDD, MDD, and the three-category depression classification (non-depressed, MiDD, and MDD; Supplementary Table S4).

\section{TABLE 4}

Bivariate Heritability Model Fits

\begin{tabular}{|c|c|c|c|c|c|}
\hline Model & $\begin{array}{l}\text { Minus } 2 \text { log } \\
\text { likelihood }\end{array}$ & $x^{2}$ & $\Delta d f$ & $p$ value & $\mathrm{AIC}$ \\
\hline $\mathrm{ACE}$ & $4,405.65$ & & & & $-5,324.35$ \\
\hline $\mathrm{AE}$ & $4,406.74$ & 1.09 & 3 & 0.78 & $-5,329.26$ \\
\hline CE & $4,419.35$ & 13.70 & 3 & $3.34 \times 10^{-3}$ & $-5,316.65$ \\
\hline $\mathrm{E}$ & $4,483.87$ & 78.22 & 6 & $8.35 \times 10^{-15}$ & $-5,258.13$ \\
\hline $\mathrm{ADE}$ & $4,405.20$ & -0.46 & 0 & 1.00 & $-5,324.80$ \\
\hline
\end{tabular}

\section{Bivariate Heritability Estimates}

Bivariate model fitting for depression and fatigue indicated that the AE model is the most parsimonious (Table 4). No differences in depression or fatigue threshold distributions were observed within twin pairs, and across zygosity and sex groups. Overall, $48 \%$ [95\% CI $=32-61 \%]$ and $41 \%$ [95\% $\mathrm{CI}=0.30-0.51 \%]$ of the variance in depression and fatigue, respectively, were explained by genetic factors. Also, $52 \%$ $[95 \% \mathrm{CI}=39-68 \%]$ and $59 \%[95 \% \mathrm{CI}=0.49-0.70 \%]$ of the variance in depression and fatigue were explained by unique environmental factors, respectively (Figure 2). Notably, $21 \%[95 \% \mathrm{CI}=10-35 \%]$ of the variance in depression due to genetic factors also contributes to the heritability of fatigue (i.e., bivariate heritability of $21 \%$ ). Also, $7 \%$ of the variance in depression due to unique environmental factors 


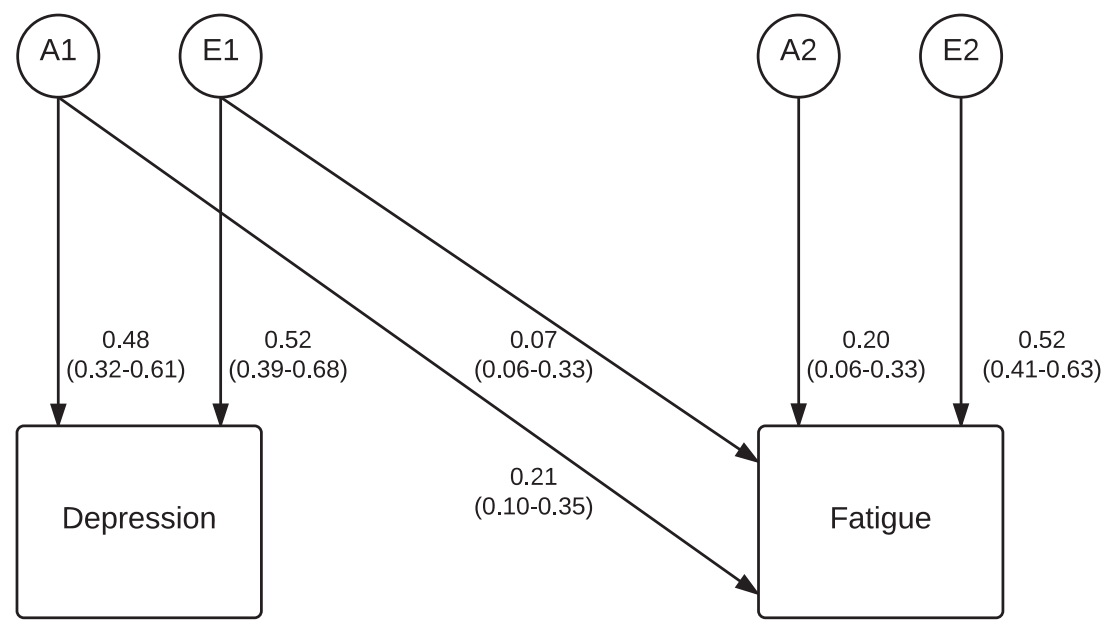

\section{FIGURE 2}

Path diagram of the bivariate Cholesky model variance estimates (with their $95 \%$ confidence intervals) for two-category depression and fatigue. The observed traits are shown in the rectangles. Similarly, the latent variables (additive genetic factors: A, and unique environmental factors: E) are depicted by circles. The arrows depict the relationship between the variables.

contributes to the variance in fatigue. The overlap in genetic and unique environmental factors was supported by $r_{\mathrm{g}}$ of $0.71[95 \% \mathrm{CI}=0.51-0.92]$ and $r_{\mathrm{e}}$ of $0.35[95 \% \mathrm{CI}=0.17-$ 0.51 ] between depression and fatigue. Similar results were obtained for bivariate heritability estimates between MDD or MiDD and fatigue (Supplementary Table S5, Supplementary Figures S1-S2). Almost identical bivariate heritability estimates were obtained between three-category depression and fatigue (Supplementary Table S5, Supplementary Figure S3). Importantly, the shared genetic contribution to depression and fatigue remained significant independent of the overlapping symptoms (Supplementary Figure S4).

\section{Co-Twin Control}

Assessment of depression as a risk factor for fatigue revealed that the ORs in the general population, discordant $\mathrm{DZ}$ twin pairs, and discordant MZ twin pairs were 7.20 [95\% CI: 4.49-11.56], 6.29 [95\% CI: 3.35-11.81], and 1.92 [95\% CI: 1.09-3.39], respectively (Figure 3). Similarly, assessment of fatigue as a risk factor for depression revealed that the ORs in the general population, discordant $\mathrm{DZ}$ twin pairs, and discordant MZ twin pairs were 7.20 [95\% CI: 4.49-11.56], 5.21 [95\% CI: 2.75-9.88], and 1.98 [95\% CI: 1.10-3.55], respectively (Figure 3). The pattern of $O R$ exhibited when fatigue was a risk factor for depression was comparable to when depression was a risk factor for fatigue. The observed $O R$ pattern indicates that a non-causal genetic model best describes the relationship between depression and fatigue. A non-causal genetic relationship was also indicated between fatigue and MiDD (Supplementary Table S6). However, the co-twin control analysis could not be replicated for fatigue and MDD due to the lack of power in the smaller subsample.
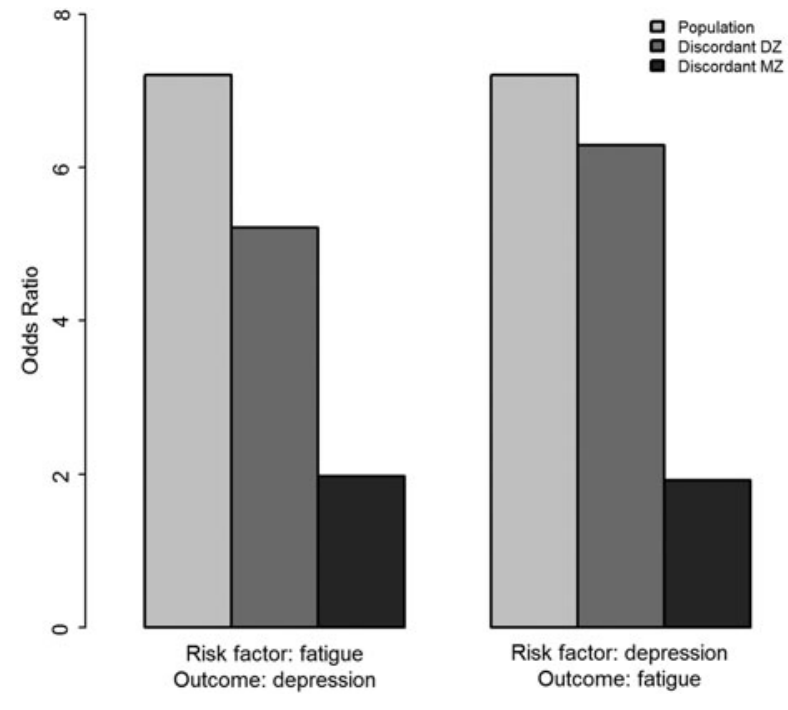

FIGURE 3

Left: The observed odds ratios (OR) for a current diagnosis of fatigue with a given a current diagnosis of depression in the general population (1,266 unrelated twin singles), 99 discordant DZ twin pairs, and 96 discordant MZ twin pairs. Right: The observed OR for a current diagnosis of depression with a given a current diagnosis of fatigue in the general population (1,266 unrelated twin singles), 200 discordant DZ twin pairs, and 215 discordant MZ twin pairs. In both situations, the observed $O R$ patterns are consistent with a non-causal genetic model.

\section{Discussion}

Three key findings were identified from the present study. First, depression and fatigue exhibit a familial component. Second, co-occurring depression and fatigue have considerable genetic overlap. Finally, a non-causal genetic model 
likely explains the association between depression and fatigue.

The familial clustering analysis revealed that depression and fatigue likely have shared underlying etiologies. These results are supported by previous studies that have reported higher levels of depression in fatigued individuals than the general population (Cathébras et al., 1992; Walker et al., 1993). However, to our knowledge, this is the first study to assess and characterize the familial clustering of depression and fatigue, with regard to the risk of depression in fatigued individuals and the risk of fatigue in depressed individuals.

Overlap in genetic factors between depression and fatigue has been indicated in previous twin studies (Ball et al., 2010; Fowler et al., 2006; Hickie et al., 1999; Hur et al., 2012). Previous multivariate twin studies have identified genetic and environmental factors that are unique to fatigue. Hur et al. (2012) established common and symptomspecific genetic and environmental factors contributed to the heritability of self-reported insomnia, fatigue, and depression experienced within a 12-month period. Similarly, Hickie et al. (1999) determined that $44 \%$ of the genetic heritability and $100 \%$ of the environmental contribution of fatigue is independent of psychological distress, anxiety, and depression. However, the proportion of genetic factors contributing to fatigue that are independent of depression appears to be smaller in older adults than in children. The bivariate modeling reported by Fowler et al. (2006) established that $87 \%$ and $73 \%$ of the genetic heritability for lifetime-ever short-duration fatigue (fatigue experienced for at least 1 week) and lifetime-ever prolonged fatigue (fatigue experienced for at least 1 month), respectively, were independent of depression within the last 3 months in children. The high proportion of genetic heritability specific to short-duration fatigue and prolonged fatigue was substantiated by $r_{\mathrm{g}}$ of 0.36 and 0.53 , respectively. However, Ball et al. (2010) indicated that unique environmental variance components explained a larger proportion of the overlap in heritability between fatigue and an indicator of lifetime depression than familial factors, in Sri Lanka.

The bivariate twin modeling results from the current study indicated $50 \%$ of the genetic heritability and $12 \%$ of the environmental contribution of fatigue are shared with depression. $r_{\mathrm{g}}$ of 0.71 between co-occurring depression and fatigue within adults (aged over 50) was considerably higher than previous bivariate studies, indicating larger genetic overlap exists between co-occurring depression and fatigue in older adults than depression within the past 3 months and lifetime-ever disabling fatigue in children. A small environmental overlap between co-occurring depression and fatigue was also observed $\left(r_{\mathrm{e}}=0.35\right)$. Differences in the contribution of genetic and environmental factors to the shared heritability of depression and fatigue between adults in Australia and Sri Lanka are likely attributable to variation in phenotypic classification, ethnicity, age, and cultural differences. In particular, the measure of depression utilized within the Sri Lankan population only assessed the two core symptoms of the DSM criteria for a major depressive episode. Therefore, the genetic correlation of depression and fatigue potentially increases with the number of depression symptoms.

Skapinakis and colleagues (2004) described four possible explanations for the association between unexplained depression and fatigue observed within their international study: the causal, reverse causality, common etiology, and overlapping criteria hypotheses. Results of the present study substantiate the common etiology hypothesis, whereby depression and fatigue share common risk factors. In particular, our results indicate that shared genetic factors explain the majority of the correlation. The larger overlap in genetic heritability between co-occurring depression and fatigue is also supported by the results of the co-twin control analysis. That is, the determined non-causal genetic relationship between depression and fatigue adds further support to the comorbidity between depression and fatigue, being primarily due to shared genetic factors.

Our findings lead us to suggest that overlapping genetic factors could also underlie the relationship between depression relapse and residual fatigue. Residual fatigue has a prevalence of $63-98 \%$ and $22-49 \%$ in partial responders and remitted patients, respectively, after antidepressant treatment (Fava et al., 2014). Additionally, fatigue as a symptom of depression has been associated with higher health care utilization, $10-20 \%$ greater annual healthcare cost, increased medication uses, and lower quality of life (Robinson et al., 2014). Furthermore, residual fatigue has been shown to lead to higher levels of functional impairment and depression relapse. Considering that currently available antidepressant therapies have been shown to inadequately treat residual fatigue, we believe research should focus on understanding the shared mechanisms of depression and fatigue. Such research holds great potential to facilitate the development of enhanced treatment outcomes, which are targeted to shared mechanisms of depression and fatigue. The effective treatment of depression focusing on symptomatic treatment of residual fatigue could lower remission levels, and thereby lower the global burden of depression.

The present study is the first to investigate the type of relationship between co-occurring depression and fatigue, utilizing a two-category and three-category depression status. Additionally, it is the only study that determined whether a causal relationship exists between depression and fatigue. A possible limitation of the study is that depression and fatigue were assessed by self-report rather than interview based. However, this allowed depression and fatigue to be assessed independently without introducing interviewer bias. Furthermore, the study was not confounded by healthcare-seeking behavior due to the population-based structure of the cohort. Additionally, the current study focused on an older age group for which some evidence 
suggests the risk of depression is similar between the sexes (Bebbington et al., 1998; Faravelli et al., 2013).

In summary, our results indicate depression and fatigue are familial, with shared genetic factors explaining a substantial proportion of the comorbidity between the traits in adults. Research focusing on the underlying pathways that are shared by depression and fatigue will facilitate the elucidation of the mechanisms driving the association.

\section{Acknowledgments}

We would like to thank the twins for their cooperation and study staff for data collection. Mr George Landers of Chania, Crete generous donations funded this research. Elizabeth C. Corfield was supported by an Australian Postgraduate Award (APA) from the Australian Government. Dale R. Nyholt was supported by an Australian National Health and Medical Research Council (NHMRC) Research Fellowship (Application ID 613674).

\section{Conflict of Interest}

None.

\section{Ethical Standards}

Informed written consent was obtained from each participant, and the study was approved by the Health Research Ethics Committee (HREC) of the QIMR Berghofer Medical Research Institute (QIMRB).

\section{Supplementary Material}

To view supplementary material for this article, please visit http://dx.doi.org/10.1017/thg.2016.79.

\section{References}

Akaike, H. (1973). Information theory and an extension of the maximum likelihood principle. Paper presented at the Second International Symposium on Information Theory, Tsahkadsor, Armenia, USSR.

Akaike, H. (1974). A new look at the statistical model identification. IEEE Transactions on Automatic Control, 19, 716723.

American Psychiatric Association. (2000). Diagnostic and statistical manual of mental disorders (4th ed.). Washington, DC: Author.

American Psychiatric Association. (2013). Diagnostic and statistical manual of mental disorders (5th ed.). Washington, DC: Author.

Ball, H. A., Sumathipala, A., Siribaddana, S. H., Kovas, Y., Glozier, N., McGuffin, P., \& Hotopf, M. (2010). Aetiology of fatigue in Sri Lanka and its overlap with depression. British Journal of Psychiatry, 197, 106-113.

Bebbington, P. E., Dunn, G., Jenkins, R., Lewis, G., Brugha, T., Farrell, M., \& Meltzer, H. (1998). The influence of age and sex on the prevalence of depressive conditions: Report from the National Survey of Psychiatric Morbidity. Psychological Medicine, 28, 9-19.

Bedford, A., \& Deary, I. J. (1997). The personal disturbance scale (DSSI/sAD): Development, use and structure. Personality and Individual Differences, 22, 493-510.

Bijl, R. V., de Graaf, R., Ravelli, A., Smit, F., \& Vollebergh, W. A. M. (2002). Gender and age-specific first incidence of DSM-III-R psychiatric disorders in the general population. Social Psychiatry and Psychiatric Epidemiology, 37, 372-379.

Boker, S., Neale, M., Maes, H., Wilde, M., Spiegel, M., Brick, T., ... Fox, J. (2011). OpenMx: An open source extended structural equation modeling framework. Psychometrika, 76, 306-317.

Bucholz, K. K., Heath, A. C., Madden, P. A. F., Slutske, W. S., Statham, D. J., Dunne, M. P., \& Martin, N. G. (1998). Drinking in an older population: Cross-sectional and longitudinal data from the Australian twin registry. In E. L. Gomberg, A. M. Hegedus, \& R. A. Zucker (Eds.), Alcohol problems and aging (pp. 41-62). Bethesda, MD: National Institutes of Health.

Cathébras, P. J., Robbins, J. M., Kirmayer, L. J., \& Hayton, B. C. (1992). Fatigue in primary care: Prevalence, psychiatric comorbidity, illness behavior, and outcome. Journal of General Internal Medicine, 7, 276-286.

Centers for Disease Control and Prevention. (2010). Current depression among adults-United States, 2006 and 2008. Morbidity and Mortality Weekly Report, 59, 1229-1235.

Chalder, T., Berelowitz, G., Pawlikowska, T., Watts, L., Wessely, S., Wright, D., \& Wallace, E. P. (1993). Development of a fatigue scale. Journal of Psychosomatic Research, 37, 147153.

Costello, E. J., \& Angold, A. (1988). Scales to assess child and adolescent depression: Checklists, screens, and nets. Journal of the American Academy of Child and Adolescent Psychiatry, 27, 726-737.

Faravelli, C., Alessandra Scarpato, M., Castellini, G., \& Lo Sauro, C. (2013). Gender differences in depression and anxiety: The role of age. Psychiatry Research, 210, 1301-1303.

Farmer, A., Scourfield, J., Martin, N., Cardno, A., \& McGuffin, P. (1999). Is disabling fatigue in childhood influenced by genes? Psychological Medicine, 29, 279-282.

Fava, M., Ball, S., Nelson, J. C., Sparks, J., Konechnik, T., Classi, P., ... Thase, M. E. (2014). Clinical relevance of fatigue as a residual symptom in major depressive disorder. Depression and Anxiety, 31, 250-257.

Fowler, T. A., Rice, F., Thapar, A., \& Farmer, A. (2006). Relationship between disabling fatigue and depression in children: Genetic study. British Journal of Psychiatry, 189, 247253.

Goldberg, D. P., \& Blackwell, B. (1970). Psychiatric illness in general practice. A detailed study using a new method of case identification. British Medical Journal, 1, 439-443.

Hickie, I., Kirk, K., \& Martin, N. (1999). Unique genetic and environmental determinants of prolonged fatigue: A twin study. Psychological Medicine, 29, 259-268.

Hickie, I. B., Hooker, A. W., HadziPavlovic, D., Bennett, B. K., \& Wilson, A. J. (1996). Fatigue in selected primary care 
settings: Sociodemographic and psychiatric correlates. Medical Journal of Australia, 164, 585-588.

Hur, Y.-M., Burri, A., \& Spector, T. D. (2012). The genetic and environmental structure of the covariation among the symptoms of insomnia, fatigue, and depression in adult females. Twin Research and Human Genetics, 15, 720-726.

Hybels, C. F., Landerman, L. R., \& Blazer, D. G. (2012). Age differences in symptom expression in patients with major depression. International Journal of Geriatric Psychiatry, 27, 601-611.

Kendler, K. S., Karkowski, L. M., \& Prescott, C. A. (1999). Causal relationship between stressful life events and the onset of major depression. American Journal of Psychiatry, 156, 837-841.

Kendler, K. S., Neale, M. C., MacLean, C. J., Heath, A. C., Eaves, L. J., \& Kessler, R. C. (1993). Smoking and major depression. A causal analysis. Archives of General Psychiatry, $50,36-43$.

Kessler, R. C., Berglund, P., Demler, O., Jin, R., Koretz, D., Merikangas, K. R., ... Wang, P. S. (2003). The epidemiology of major depressive disorder: Results from the National Comorbidity Survey Replication (NCS-R). JAMA, 289, 30953105.

Ligthart, L., Nyholt, D. R., Penninx, B. W. J. H., \& Boomsma, D. I. (2010). The shared genetics of migraine and anxious depression. Headache: The Journal of Head and Face Pain, 50, 1549-1560.

McGue, M., Osler, M., \& Christensen, K. (2010). Causal inference and observational research: The utility of twins. Perspectives on Psychological Science, 5, 546-556.

Mosing, M. A., Medland, S. E., McRae, A., Landers, J. G., Wright, M. J., \& Martin, N. G. (2012). Genetic influences on life span and its relationship to personality: A 16-year follow-up study of a sample of aging twins. Psychosomatic Medicine, 74, 16-22.

Neale, M. C., \& Cardon, L. R. (1992). Methodology for genetic studies of twins and families. New York, NY: Springer.
Nyholt, D. R., Gillespie, N. G., Heath, A. C., Merikangas, K. R., Duffy, D. L., \& Martin, N. G. (2004). Latent class and genetic analysis does not support migraine with aura and migraine without aura as separate entities. Genetic Epidemiology, 26, 231-244.

R Core Team. (2014). R: A language and environment for statistical computing. Vienna, Austria: R Foundation for Statistical Computing. Retrieved from http://www.R-project.org/.

Robinson, R. L., Stephenson, J. J., Dennehy, E. B., Grabner, M., Faries, D., Palli, S. R., \& Swindle, R. W. (2014). The importance of unresolved fatigue in depression: Costs and comorbidities. Psychosomatics, 56, 274-285.

Schur, E., Afari, N., Goldberg, J., Buchwald, D., \& Sullivan, P. F. (2007). Twin analyses of fatigue. Twin Research and Human Genetics, 10, 729-733.

Skapinakis, P., Lewis, G., \& Mavreas, V. (2004). Temporal relations between unexplained fatigue and depression: Longitudinal data from an international study in primary care. Psychosomatic Medicine, 66, 330-335.

Sullivan, P. F., Evengard, B., Jacks, A., \& Pedersen, N. L. (2005). Twin analyses of chronic fatigue in a Swedish national sample. Psychological Medicine, 35, 1327-1336.

Sullivan, P. F., Neale, M. C., \& Kendler, K. S. (2000). Genetic epidemiology of major depression: Review and meta-analysis. American Journal of Psychiatry, 157, 1552-1562.

Walker, E. A., Katon, W. J., \& Jemelka, R. P. (1993). Psychiatric disorders and medical care utilization among people in the general population who report fatigue. Journal of General Internal Medicine, 8, 436-440.

Wilkowska-Chmielewska, J., Szelenberger, W., \& Wojnar, M. (2013). Age-dependent symptomatology of depression in hospitalized patients and its implications for DSM-5. Journal of Affective Disorders, 150, 142-145.

World Health Organization. (1990). Composite international diagnostic interview (CIDI). Geneva, Switzerland: World Health Organization Division of Mental Health. 